\title{
CALCULATIONS OF EuTe MAGNETIC PHASE DIAGRAM FOR EXTERNAL PRESSURE
}

\author{
A. RADOMSKa AND T. BALCERZAK* \\ Solid State Physics Department, University of Łódź \\ Pomorska 149/153, 90-236 Eódź, Poland \\ (Received February 23, 2000; in final form April 28, 2000)
}

The magnetic phase diagram of europium telluride, EuTe, under external high pressure is theoretically investigated. Besides nearest-neighbour and next-nearest-neighbour exchange interactions, the long-range dipolar and biquadratic interactions are taken into account. The functional dependences of these interactions on the lattice constant are proposed. The calculated Néel and Curie temperatures are compared with the experimental data. Two new phases, antiferrimagnetic and ferrimagnetic, have been found at a high pressure and low temperatures.

PACS numbers: 75.10.-b, 75.50.Ee, 75.50.Pp

\section{Introduction}

In the group of europium chalcogenides $\operatorname{EuX}(\mathrm{X}=0, \mathrm{~S}, \mathrm{Se}, \mathrm{Te})$ the europium telluride, EuTe, is a well-known example of second-type antiferromagnet [1-5]. EuTe crystallizes in the cubic $\mathrm{NaCl}$ lattice and its magnetic structure is of the $\mathrm{MnO}$ type. The spontaneous magnetisation favours antiparallelly alignment in (111) planes with a small anisotropy along $\langle 11 \overline{2}\rangle$ directions [4].

The magnetic properties of EuTe arise from well-localized spins of $\mathrm{Eu}^{++}$ ions. The $\mathrm{Eu}^{++}$ion has the ${ }^{8} S_{7 / 2}$ ground state and a weak interaction with the crystalline field. Being a semiconductor, EuTe is a good example of Heisenberg isotropic magnet with the spin magnitude $S=7 / 2$ [4-6]. Its former theoretical description has been given within the Heisenberg model with nearest-neighbour (nn) and next-nearest-neighbour ( $\mathrm{nnn}$ ) interactions taken into account [5]. The number of $\mathrm{nn}$ is $z_{1}=12$, with 6 spins laying in the same (111) plane and with 6 spins in the neighbouring (antiparallelly polarized) planes. The number of nnn is $z_{2}=6$, and in this case all spins are laying in the neighbouring planes.

At a normal pressure, the $\mathrm{nn}$ direct interaction, $J_{1}$, is known to be positive and about three or four times weaker than the nnn one, $J_{2}$, which is indirect (via

\footnotetext{
*corresponding author, e-mail: tbalcerz@krysia.uni.lodz.pl
} 
chalcogen ligand) and negative. The resulting spontaneous antiferromagnetic state exists below the Néel temperature $T_{N} \approx 9.6 \mathrm{~K}$. The asymptotic Curie temperature is known approximately as $T_{\mathrm{C}} \approx-4 \mathrm{~K}$ [5]. The lattice constant of EuTe at a normal pressure is $a_{0}=6.598 \AA$ and the magnetic unit cell is twice bigger than the chemical unit cell.

The first theoretical description of EuTe was done in the molecular field approximation (MFA) for a Heisenberg magnet, with only $J_{1}$ and $J_{2}$ interactions taken into account [5]. However, as it was shown in [7] by the energy arguments, the long-range dipolar interactions are also important, being responsible for the spins alignment within the (111) plane. The value of dipolar interaction was estimated in [7] and its dependence on the lattice constant $\left(\sim a^{-3}\right)$ was there established.

Moreover, in the papers $[8,9]$ the role of biquadratic interactions was pointed out. These interactions enabled us to explain the magnetisation measurements versus temperature [8], and were also discussed in the context of the Curie temperature and susceptibility calculations [9]. Thus, the presence of biquadratic interactions can help in explaining some experimental details which could not be predicted within the pure Heisenberg model.

Apart from one single attempt [10], the last two above-mentioned interactions, i.e., dipolar and biquadratic, have never been taken into account simultaneously together with the Heisenberg Hamiltonian. In this paper we intend to improve this deficiency, at least in the case of the description of EuTe.

The increasing interest in studies of EuTe is connected with the measurements under high pressure [11-14]. The magnetic phase diagram of EuTe has been found in [13] for a different external pressure and lattice constant $a$ corresponding to it. It turned out that near $8 \mathrm{GPa}$ EuTe undergoes a pressure-induced phase transition from antiferromagnetic to ferromagnetic ordering. The ferromagnetic state can exist until about $11.7 \mathrm{GPa}$ where the structural change to $\mathrm{CsCl}$ lattice takes place [11].

The aim of the present paper is the theoretical explanation of the EuTe phase diagram obtained in [13]. Apart from the classical Heisenberg Hamiltonian with nn and nnn interactions, we will take into account the dipolar and biquadratic interactions as well. As the external pressure changes the lattice constant, $a$, we have to take into account the lattice constant dependences of all relevant interactions. Such dependences are generally unknown from the theory (with the exception of dipolar interactions) although some suggestions concerning $J_{1}(a)$ and $J_{2}(a)$ can be found in the literature $[2,5,6,14]$. However, it turns out from our calculations that, if the biquadratic interactions are taken into account, the former assumptions about $J_{1}(a)$ and $J_{2}(a)$ have to be essentially verified.

For the studies we adopted the molecular field approximation based on the Gibbs free energy analysis. The theoretical method is developed in the next section. In the third section the numerical results are presented and their discussion has been made. 


\section{Theory}

The Hamiltonian of the system can be written in the following form:

$$
\begin{aligned}
\mathcal{H}= & -J_{1} \sum_{\mathrm{nn}} S_{i} S_{j}-J_{2} \sum_{\mathrm{nnn}} S_{i} S_{j}-A \sum_{\substack{\mathrm{nn} \\
\mathrm{nnn}}}\left(S_{i} S_{j}\right)^{2}+g \mu_{\mathrm{B}} H^{z} \sum_{i} S_{i}^{z} \\
& +\frac{\left(g \mu_{\mathrm{B}}\right)^{2}}{2} \sum_{i, j} \frac{1}{r_{i, j}^{3}}\left[S_{i} \boldsymbol{S}_{j}-3 \frac{\left(\boldsymbol{S}_{i} \boldsymbol{r}_{i, j}\right) \cdot\left(\boldsymbol{S}_{j} \boldsymbol{r}_{i, j}\right)}{r_{i, j}^{2}}\right],
\end{aligned}
$$

where the spin magnitude $S=7 / 2, J_{1}$ and $J_{2}$ are the exchange integrals for $\mathrm{nn}$ and nnn interactions, respectively, whereas $A$ describes the strength of biquadratic interactions, both $\mathrm{nn}$ and $\mathrm{nnn}$. The fourth term in the Hamiltonian stands for the interaction with an external field, and the last term describes the long-range dipolar interactions.

The Gibbs energy of the system can be calculated for the Hamiltonian (1) from the well-known Bogolyubov expression

$$
G=G\left(\mathcal{H}_{0}\right)+\left\langle\mathcal{H}-\mathcal{H}_{0}\right\rangle_{0}
$$

In Eq. (2) $\langle\ldots\rangle_{0}$ means the thermodynamic mean value calculated with the density matrix corresponding to some trial Hamiltonian $\mathcal{H}_{0}$. Here we assume that the trial Hamiltonian is essentially the molecular field Hamiltonian, containing as the variational parameters the molecular fields associated with the bilinear and biquadratic interactions. Performing a minimisation procedure for the functional (2) with respect to the variational parameters, the equilibrium Gibbs energy per spin can be found in the following form:

$$
\begin{aligned}
\frac{G}{N}= & -\frac{1}{2} k_{\mathrm{B}} T \ln Z_{a}-\frac{1}{2} k_{\mathrm{B}} T \ln Z_{b}+\frac{1}{2}\left(\frac{z_{1}}{2} J_{1}-\alpha\right)\left(m_{a}^{2}+m_{b}^{2}\right) \\
& +\left(\frac{z_{1}}{2} J_{1}+z_{2} J_{2}\right) m_{a} m_{b}+\frac{z_{1}}{4} A\left(q_{a}^{2}+q_{b}^{2}\right)+\left(\frac{z_{1}}{2}+z_{2}\right) A q_{a} q_{b} .
\end{aligned}
$$

In Eq. (3) we introduce the following notation: $m_{i}=\left\langle S_{i}\right\rangle_{0}$ and $q_{i}=\left\langle\left(S_{i}\right)^{2}\right\rangle_{0}$ are the magnetisations and quadrupolar moments per spin in $i$-th (111) plane, respectively. The index $i=a, b$, where $a$ and $b$ are the two neighbouring (111) planes of the antiferromagnet. The magnetisations $m_{i}$ and quadrupolar moments $q_{i}$ are then given by the analytical formulae

$$
\begin{aligned}
& m_{i}=\frac{M_{i}}{Z_{i}}, \\
& q_{i}=\frac{Q_{i}}{Z_{i}} \quad(i=a, b),
\end{aligned}
$$

where

$$
\begin{aligned}
& Z_{i}=2 \sum_{n=0}^{3} \cosh \left(\frac{2 n+1}{2} \beta x_{i}\right) \exp \left[\frac{(2 n+1)^{2}}{4} \beta y_{i}\right] \\
& M_{i}=\sum_{n=0}^{3}(2 n+1) \sinh \left(\frac{2 n+1}{2} \beta x_{i}\right) \exp \left[\frac{(2 n+1)^{2}}{4} \beta y_{i}\right]
\end{aligned}
$$


and

$$
Q_{i}=\frac{1}{2} \sum_{n=0}^{3}(2 n+1)^{2} \cosh \left(\frac{2 n+1}{2} \beta x_{i}\right) \exp \left[\frac{(2 n+1)^{2}}{4} \beta y_{i}\right] \text {. }
$$

In Eqs. (6-8), the following abbreviated notation was used:

$$
\begin{aligned}
& x_{a}=2\left(\frac{z_{1}}{2} J_{1}-\alpha\right) m_{a}+2\left(\frac{z_{1}}{2} J_{1}+z_{2} J_{2}\right) m_{b}+h, \\
& x_{b}=2\left(\frac{z_{1}}{2} J_{1}-\alpha\right) m_{b}+2\left(\frac{z_{1}}{2} J_{1}+z_{2} J_{2}\right) m_{a}+h
\end{aligned}
$$

and

$$
\begin{aligned}
& y_{a}=z_{1} A q_{a}+2\left(\frac{z_{1}}{2}+z_{2}\right) A q_{b}, \\
& y_{b}=z_{1} A q_{b}+2\left(\frac{z_{1}}{2}+z_{2}\right) A q_{a}
\end{aligned}
$$

with $\beta=1 / k_{\mathrm{B}} T$ and $h=-g \mu_{\mathrm{B}} H^{z}$. The $\alpha$ parameter appearing in the above equations corresponds to the long-range dipolar interactions and is given by the formula

$$
\alpha=\frac{\left(g \mu_{\mathrm{B}}\right)^{2}}{2} \sum_{j} \frac{1}{r_{i, j}^{3}}\left[1-3\left(\frac{z_{i, j}}{r_{i, j}}\right)^{2}\right],
$$

where $r_{i, j}$ is the distance between $i$-th and $j$-th spin and $z_{i, j}$ is a projection of $r_{i, j}$ on the magnetisation ( $z$-axis).

Thus, we see that in order to calculate the Gibbs energy from Eq. (3) one has first to solve four coupled equations (4) and (5), for $m_{i}$ and $q_{i}(i=a, b)$.

Having calculated the Gibbs energy, one can obtain all thermodynamic (magnetic) properties of the system in equilibrium. For instance, the sublattice magnetisations $m_{i}(i=a, b)$ satisfy the following thermodynamic formula:

$$
\frac{1}{2}\left(m_{a}+m_{b}\right)=-\frac{1}{N}\left(\frac{\partial G}{\partial h}\right)_{T}
$$

giving the mean magnetisation per spin in the system.

By linearisation of the magnetisation (i.e., $m_{a} \rightarrow 0$ and $m_{b} \rightarrow 0$ in Eqs. (4) and (5)) and putting $q_{a}=q_{b}=q$, we obtain the temperature of continuous phase transitions from an ordered to a paramagnetic state. The result is

$$
k_{\mathrm{B}} T_{\mathrm{C}, \mathrm{N}}=2 q\left[\frac{z_{1}}{2} J_{1}-\alpha \pm\left(\frac{z_{1}}{2} J_{1}+z_{2} J_{2}\right)\right] \text {, }
$$

where "-" corresponds to the Néel temperature $T_{\mathrm{N}}$ and "+" corresponds to the Curie temperature $T_{\mathrm{C}}$. The quadrupolar moment $q$ is then given by the formula

$$
q=\frac{1}{4}\left\{\sum_{n=0}^{3}(2 n+1)^{2} \exp \left[\frac{(2 n+1)^{2}}{4} r\right]\right\} /\left\{\sum_{n=0}^{3} \exp \left[\frac{(2 n+1)^{2}}{4} r\right]\right\}
$$

where

$$
r=\left(z_{1}+z_{2}\right) A /\left[\frac{z_{1}}{2} J_{1}-\alpha \pm\left(\frac{z_{1}}{2} J_{1}+z_{2} J_{2}\right)\right] .
$$

Again, the signs "-" and "+" in Eq. (17) correspond to $T_{\mathrm{N}}$ and $T_{\mathrm{C}}$, respectively. 
It is straightforward to check from Eq. (15) that if we neglect the dipolar interactions (i.e., $\alpha=0)$ and biquadratic interactions $(A=0)$ then our formula (15) gives the well-known expressions for the Neel and Curie temperatures of $S=7 / 2$ magnet, with $z_{1}=12$ and $z_{2}=6[2,4-6]$ namely:

$$
k_{\mathrm{B}} T_{\mathrm{N}}=-63 J_{2}
$$

and

$$
k_{\mathrm{B}} T_{\mathrm{C}}=126 J_{1}+63 J_{2} .
$$

Thus, our formula (15) for the continuous (second order) phase transitions is more general than those (18) and (19) known from the literature, taking into account the dipolar and biquadratic interactions. Besides, the expression (3) for the Gibbs energy allows us also to study the possible first order phase transitions, where the linearisation of magnetisation is not valid. The numerical results based on the above theoretical formulae will be presented in the next section.

\section{The numerical results and discussion}

The first calculations of the exchange integrals $J_{1}$ and $J_{2}$ were done in the literature at a normal pressure on the basis of Eqs. (18) and (19), i.e., with the long-range dipolar and biquadratic interactions neglected. On the basis of the experimental data for $T_{\mathrm{N}}$ and $T_{\mathrm{C}}$, the obtained values for $J_{1}$ and $J_{2}$ were averaged and presented in [5]. For EuTe the corresponding values are [5]: $J_{1} / k_{\mathrm{B}}=$ $(0.06 \pm 0.02) \mathrm{K}$ and $J_{2} / k_{\mathrm{B}}=(-0.20 \pm 0.05) \mathrm{K}$. As it has been shown in [10], the presence of the long-range dipolar and biquadratic interactions may markedly change the values of $J_{1}$ and $J_{2}$. Namely, the strength of dipolar interactions (given by Eq. (13)) can be estimated as [7]

$$
\alpha=-\mu_{\mathrm{B}}^{2} \frac{11.035}{(a / \sqrt{2})^{3}}
$$

where $g \approx 2$ and $a$ is the lattice constant of EuTe expressed in $\AA$.

On the other hand, the biquadratic interaction strength at a normal pressure can be assumed as suggested in the paper [8], namely $A / J_{2}=1.5 \times 10^{-3}$. Having these two results and assuming that $T_{\mathrm{N}} \approx 9.6 \mathrm{~K}$ and $T_{\mathrm{C}} \approx-4 \mathrm{~K}$, we obtained the new values for $J_{1}$ and $J_{2}[10]: J_{1} / k_{\mathrm{B}}=0.036 \mathrm{~K}$ and $J_{2} / k_{\mathrm{B}}=-0.144 \mathrm{~K}$. These values will be now a starting point for further considerations including an external pressure.

Under an external pressure the lattice constant $a$ diminishes from $a=a_{0}=$ $6.598 \AA$ (at $p=0$ ) until $a \approx 6.21 \AA$ (at $p=11.7 \mathrm{GPa}$ ), as presented in [13]. The change of the long-range dipolar interactions vs. $a$ can be obtained from formula (20). In Fig. 1 we plotted this dependence, showing that for higher pressures the absolute value of the long-range dipolar parameter $\left(\alpha / k_{\mathrm{B}}\right)$ increases, and thus these interactions become more important.

As far as exchange integrals are concerned, we assumed that they can be described by the exponential functions of the type

$$
J_{1,2}(a)=B_{1,2} \exp \left[C_{1,2}\left(a-D_{1,2}\right)^{2}\right] .
$$




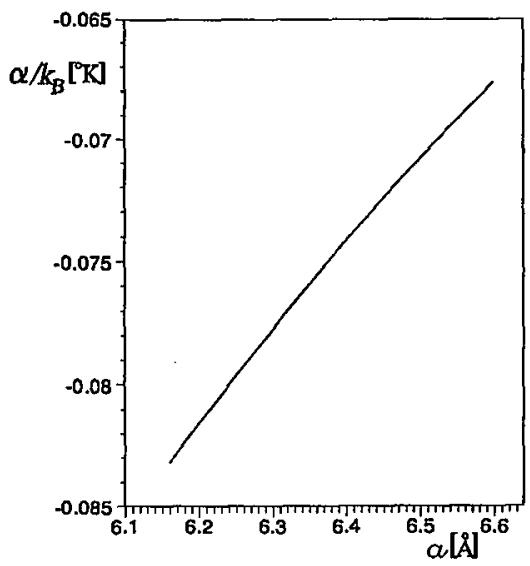

Fig. 1. The long-range dipolar interaction parameter $\alpha / k_{\mathrm{B}}$ vs. lattice constant $a$.

The three constant parameters for each function $J_{1}$ and $J_{2}$, i.e., $B_{1,2}, C_{1,2}$, and $D_{1,2}$, were obtained from three experimental points for $T_{\mathrm{N}}$ and $T_{\mathrm{C}}$ at different pressures. We have assumed the following experimental data [13]: at $p=1$ atm, $a=a_{0}=6.598 \AA, T_{\mathrm{N}}=9.6 \mathrm{~K}, T_{\mathrm{C}}=-4 \mathrm{~K}$; at $p=4 \mathrm{GPa}, a=6.415 \AA$, $T_{\mathrm{N}}=10.6 \mathrm{~K}, T_{\mathrm{C}}=1 \mathrm{~K}$; at $p=8 \mathrm{GPa}, a=6.29 \AA, T_{\mathrm{N}}=T_{\mathrm{C}}=8.7 \mathrm{~K}$. One should note that the values of $T_{\mathrm{C}}$ for $p=1 \mathrm{~atm}$ and $p=4 \mathrm{GPa}$ are the so-called "asymptotic" Curie temperatures, whereas $T_{\mathrm{C}}$ for $p=8 \mathrm{GPa}$ is a real phase transition temperature. The asymptotic Curie temperatures have been taken from the susceptibility measurements (Fig. 3 of Ref. [13]). On the other hand, the real Néel and Curie temperatures have been shown in Fig. 5 of Ref. [13]. Using these values we can calculate 6 coefficients $B_{1,2}, C_{1,2}$, and $D_{1,2}$ from Eq. (15), provided the biquadratic interactions in these points are known.

On the other hand, for the biquadratic interactions (both nn and nnn) we assumed the two-parameter exponential curve

$$
A(a) / J_{2}(a)=E \exp \left[-F\left(a-a_{0}\right)\right] .
$$

The $E$ parameter in (22) is known from the literature, since it can be defined at a normal pressure, when $a=a_{0}$. Then, to be consistent with the previous results $[8,10]$ we must assume that $E=0.0015$. The last parameter in Eq. (22), i.e., $F$, has been obtained from the assumption that the total phase diagram must be best fitted to the experimental data. To achieve this task, we took into account all experimental data presented in the phase diagram [13]. Then, we calculated a series of phase diagrams, each for different $F$ parameter. For each phase diagram we found the least squares coefficient $\mathcal{E}$ defined as

$$
\mathcal{E}=\sum_{k}^{\substack{\text { all } \\ \text { data }}}\left[\frac{T_{\mathrm{N}, \mathrm{C}}^{\exp }(k)-T_{\mathrm{N}, \mathrm{C}}^{\text {theor. }}(k)}{T_{\mathrm{N}, \mathrm{C}}^{\exp }(k)}\right]^{2} .
$$

For the smallest coefficient $\mathcal{E}(\mathcal{E}=0.012)$ we found the best value of $F$ parame- 
ter $(F=13.5)$, and hence all remaining parameters in $(21)$ and (22) have been obtained. The final results are

$$
J_{1} / k_{\mathrm{B}}=0.0264 \exp \left[12.710(a-6.753)^{2}\right]
$$

for $\mathrm{nn}$ interactions, and

$$
J_{2} / k_{\mathrm{B}}=-0.1420 \exp \left[13.378(a-6.569)^{2}\right]
$$

for nnn interactions. Thus, for the biquadratic interactions we obtained the following function:

$$
A(a) / J_{2}(a)=0.0015 \exp \left[-13.500\left(a-a_{0}\right)\right] .
$$

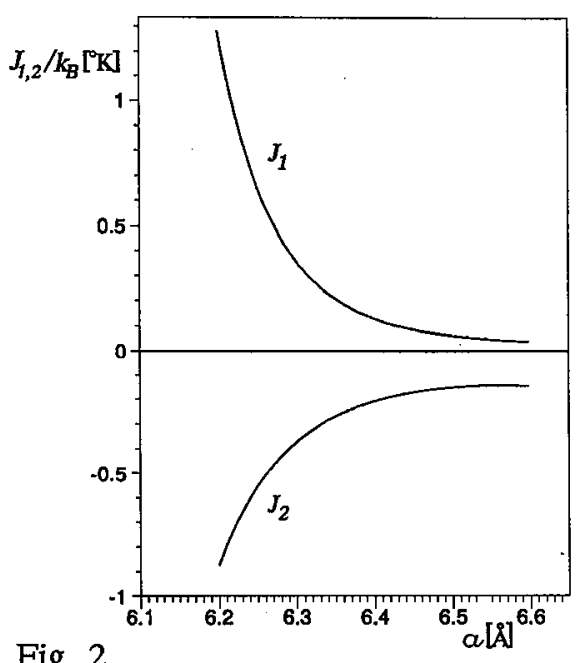

Fig. 2

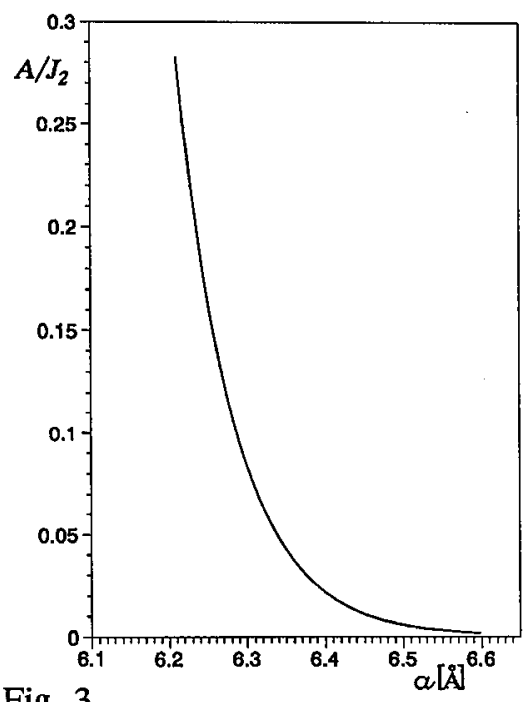

Fig. 3

Fig. 2. The nn exchange interaction $J_{1}$ and nnn exchange interaction $J_{2}$ vs. lattice constant $a$.

Fig. 3. The ratio of biquadratic to $n n n$ interactions, $A / J_{2}$, vs. lattice constant $a$.

The results of these calculations are presented in Figs. 2 and 3. From Fig. 2 we see the strong dependence of both $J_{1}(a)$ and $J_{2}(a)$ on the lattice constant. The character of $J_{1}(a)$ dependence agrees qualitatively with that reported in $[2,5,6]$, obtained from EuX series. However, the $J_{2}(a)$ dependence shows rather a different behaviour. It is not a constant, as assumed in $[2,6]$ from $\mathrm{EuX}$ series, neither a decreasing function of $a$, as found in [5]. The shapes of $J_{1}(a)$ and $J_{2}(a)$ were also discussed in [14]. The authors of [14] came to the conclusion that the functional dependences of $J_{1}(a)$ and $J_{2}(a)$ obtained from the whole series of europium monochalcogenides cannot be adopted for the description of pressure dependence of one compound. In the light of our calculations we fully agree with the conclusion that each compound should be treated separately.

The exponential function $A / J_{2}$ is presented in Fig. 3. We see the rapid increase in $A / J_{2}$ for high pressures (at low lattice constant values) although the value 
of $A$ remains always less than $J_{2}$. To check the influence of biquadratic interactions on the phase diagram we performed also calculations with the assumption that $A=0$, instead of Eq. (22). Then, from the least squares fitting we obtained the coefficient $\mathcal{E}=0.077$ for the entire phase diagram, which is a much worse result than the value $\mathcal{E}=0.012$ obtained with the function (26). The worse result for $A=0$ originates mainly from the underestimation of the Curie temperatures in a ferromagnetic phase. Thus, it becomes evident that the biquadratic interactions cannot be neglected in the phase diagram calculations.

Finally, the best fitted phase diagram of EuTe is presented in Fig. 4. The solid curves are representing the continuous phase transitions from antiferromagnetic (AF) and ferromagnetic (F) to paramagnetic (P) state, i.e., the Néel $\left(T_{N}\right)$ and Curie $\left(T_{\mathrm{C}}\right)$ temperatures, respectively. These curves were calculated from the expression (15) with the help of (20) and (24-26). For the comparison, the experimental data taken from [13] are presented in the same figure. One can see that the theoretical curves are satisfactorily fitting the experimental data. In particular, the smooth experimental maximum of $T_{\mathrm{N}}$ occurring near $4 \mathrm{GPa}$ can be obtained if we assume that $J_{2}(a)$ is an exponential function. This maximum could not be explained if $J_{2}(a)$ were a linear function of the lattice constant [13].

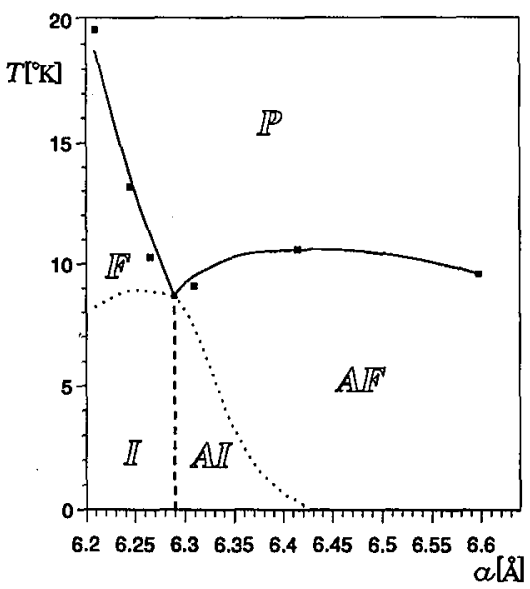

Fig. 4. The phase diagram calculated for EuTe. The small squares represent the experimental data taken from [13]. The regions of stability of various phases are depicted.

In this diagram, however, for lower temperatures and high pressures we found two new phases, i.e., the antiferrimagnetic (AI) and ferrimagnetic (I) ones, which have not been reported in the previous papers. Both these phases are characterized by different sublattice magnetisations $\left(m_{a} \neq m_{b}\right)$ and different quadrupolar moments $\left(0<q_{a} \neq q_{b}>0\right)$, where $a$ and $b$ are two neighbouring (111) planes. For the I-phase both magnetisations are of the same sign as they emerge from the ferromagnetic phase. On the other hand, the AI-phase emerges from the antiferromagnetic phase, and therefore is characterized by the opposite signs of $m_{a}$ and $m_{b}$. 
The stability of AI and I phases has been confirmed by the Gibbs energy analysis (Eqs. (3)), when the various possible solutions of Eqs. (4) and (5) were taken into account. The phase transition lines (dotted) from AI to AF phase and from I to $\mathrm{F}$ are the continuous phase transitions. The vertical line (dashed) between the I and AI phases represents the boundary of discontinuous (first order) phase transitions, and is determined by the crossing of the Gibbs energies of neighbouring phases.

The new obtained phases, i.e., AI and I, are resulting from the strong biquadratic interactions in the high pressure region, as $A$ influences there the competition between $J_{1}$ and $J_{2}$. The biquadratic interactions, having a negative sign, suppress the higher states of the spin $S=7 / 2$ and therefore they lower the ground state magnetisation, when $T \rightarrow 0$.

In conclusion, we calculated the phase diagram of EuTe under the high pressure, describing the experimental data [13]. The long-range dipolar and short-range biquadratic interactions were taken into account. The analytical, semi-empirical formulas for $J_{1,2}(a)$ and $A(a)$ have been found. In addition, the new AI and I phases have been predicted to occur in some region of the phase diagram. The experimental verification whether the AI and I phases do exist or not in EuTe would be a crucial test to check the reasonableness of our assumption about the strength of biquadratic interactions in this compound.

\section{Acknowledgments}

The authors are grateful to Dr. Mamoru Ishizuka from Osaka University for making accessible the experimental data and valuable discussions.

\section{References}

[1] S. Van Houten, Phys. Lett. 2, 215 (1962).

[2] T.R. McGuire, B.E. Argyle, M.W. Shafer, J.S. Smart, J. Appl. Phys. 34, 1345 (1963).

[3] G. Will, S.J. Pickart, H.A. Alperin, R. Nathans, J. Phys. Chem. Solids 24, 1679 (1963).

[4] N.F. Oliveira Jr., S. Foner, Y. Shapira, T.B. Reed, Phys. Rev. B 5, 2634 (1972).

[5] W. Zinn, J. Magn. Magn. Mater. 3, 23 (1976).

[6] W.P. Wolf, T.R. McGuire, M.W. Shafer, J. Appl. Phys. 35, 984 (1964).

[7] L. Bergomi, J.J. Chen, Phys. Rev. B 56, 3281 (1997).

[8] J.J. Chen, G. Dresselhaus, M.S. Dresselhaus, G. Springholz, C. Pichler, G. Bauer, Phys. Rev. B 54, 402 (1996).

[9] U. Köbler, R. Mueller, L. Smardz, D. Maier, K. Fischer, B. Olefs, W. Zinn, Z. Phys. B 100, 497 (1996).

[10] T. Balcerzak, A. Radomska, Acta Phys. Pol, A 97, 775 (2000).

[11] A. Chatterjee, A.K. Singh, A. Jayaraman, Phys. Rev. B 6, 2285 (1972).

[12] U.F. Klein, J. Moser, G. Wortmann, G.M. Kalvius, Physica B 86-88, 118 (1977).

[13] M. Ishizuka, Y. Kai, R. Akimoto, M. Kobayashi, K. Amaya, S. Endo, J. Magn. Magn. Mater. 166, 211 (1997).

[14] I.N. Goncharenko, I. Mirebeau, Phys. Rev. Lett. 80, 1082 (1998). 